\title{
CURVELET BASED SATELLITE IMAGE NATURAL RESOURCE CLASSIFICATION SYSTEM USING ELM
}

\author{
Anita Dixit ${ }^{1}$, Nagaratna P. Hegde ${ }^{2}$ and B. Eswara Reddy ${ }^{3}$ \\ ${ }^{1}$ Department of Computer Science, Jawaharlal Nehru Technological University, Anantapur, India \\ ${ }^{2}$ Department of Computer Science, Vasavi College of Engineering, India \\ ${ }^{3}$ Department of Computer Science, JNTUA College of Engineering, India
}

\begin{abstract}
Remote sensing is one of the hottest topics of research, which intends to study or analyze a particular object in the topographic map. The monitoring and management is possible when it is possible to differentiate the objects in the satellite image. However, satellite image classification is not easy, as it consists of numerous minute details. In addition to this, the accuracy and faster execution of the classification system are significant factors. This article presents a satellite image classification system that is capable of differentiating between soil, vegetation and water bodies. To achieve the goal, we categorize the entire system into three major phases; they are satellite image preprocessing, feature extraction and classification. The initial phase attempts to denoise the satellite image by the adaptive median filter and the contrast enhancement is done by Contrast Limited Adaptive Histogram Equalization (CLAHE). As the satellite image possess many important features, this work extracts curvelet moments by applying curvelet transform. The feature vector is formed out of these curvelet moments and the ELM classifier is used to train these features. The performance of the proposed approach is observed to be satisfactory in terms of sensitivity, specificity, and accuracy.
\end{abstract}

Keywords:

Remote Sensing, Satellite Image Classification, Feature Extraction

\section{INTRODUCTION}

Remote sensing is one of the active research areas, which intends to check or detect certain earthly objects. The sensing equipment of this technology are very powerful to grasp all the minute details. This technology can give a clear-cut picture of any object on the earth. Understanding the potential of this technology, several real-time applications use remote sensing to perform analysis, detection and classification. Remote sensing applications are employed in domains such as geography, oceanography, defense, ecology and so on.

Remote sensing satellites are used to monitor these objects of interest. The satellite images provide intricate details about the object. However, it is complicated to classify between all the objects being present in certain geographic area. For most of the applications, such as to locate the land use and land cover, locate the kind of vegetation in certain geographic area, it is necessary to differentiate between the objects or natural resources

This paper presents a natural resource classification system based on texture features. In this paper, the term 'natural resource classification' refers to the classification of vegetation, soil and water bodies. In order to achieve the research goal, the images that contain all the three natural resources (vegetation, soil and water bodies) are chosen as the input images. The whole work is subdivided into three vital phases, which are, image preprocessing, feature extraction and classification.
The image pre-processing phase prepares the image for the forthcoming phases. This makes sense that the pre-processing step makes up the input images by removing the noise and enhancing the quality of an image. In this work, the image pre-processing is done by adaptive median filter and the contrast of the images is improved by Contrast Limited Adaptive Histogram Equalization (CLAHE) technique. The pre-processing step is followed by the feature extraction phase. The texture features of the satellite images are extracted by applying curvelet transform. Finally, Extreme Learning Machine (ELM) is employed as the classifier to distinguish between soil, vegetation and water bodies. Some of the major features of this work are listed below

- The utilization of adaptive median filter denoises and conserves the edges of the images as well.

- The feature set is extracted from the input images by means of curvelet moments.

- The classification phase is achieved by ELM, as ELM learning is fast and efficient

The rest of this article is systematized as follows. The review of literature with respect to image classification is presented in section 2. The proposed approach to classify between soil, vegetation and water bodies is elaborated in section 3 . Section 4 analyzes the performance of the proposed approach by employing several standard performance metrics. The concluding remarks are presented in section 5 .

\section{REVIEW OF LITERATURE}

This section presents the related review of literature with respect to satellite image classification. In the literature, there are numerous satellite image classification techniques. Most of the existing satellite image classification techniques incorporate the distance measures, non-parametric classifiers, machine learning techniques and so on [1-3]. These classification algorithms utilize sufficient reference information for the purpose of training. The training stage enables the classifier to gain knowledge and helps it to differentiate between the images in the testing stage. The image classification can be achieved by pixel based classification techniques, knowledge based classification, hybrid of multiple classifiers, texture features, image transformation techniques and so on.

The basic form of classification is done by pixel based classifiers and some of the eminent pixel based algorithms are decision tree classifier, k-NN, SVM and so on. The work presented in [4] proposes a satellite image classification technique based on fuzzy logic and the work concluded that the classification accuracy is high. In [5], a satellite image classification method to differentiate between water, urban and green land is proposed. This work utilizes k-means and Latent 
Dirichlet Allocation techniques for achieving the goal. A decision tree learning based supervised satellite image classification technique is proposed in [6]. This technique extracts the features by taking the colour and intensity into account and the image classification is attained by decision tree. The work proposed in [7] utilizes a Bayesian technique based satellite image classification technique. This work exploits the spatial properties of a satellite image to distinguish between the objects present in an image. The Bayesian classifiers are trained with the texture and the spatial features of an image. This is followed by the conversion of the pixel level information to regions. Motivated by the above works, this paper aims to present a satellite image classification system for randomly selected images from Quickbird [8]. The proposed technique employs ELM as the classifier for the purpose of distinguishing between the land, vegetation and water sources. This paper extracts curvelet moments from the images. The feature vector is constructed by combining these features and the ELM is trained. The proposed approach is elaborated in the following section.

\section{PROPOSED NATURAL RESOURCE CLASSIFICATION SYSTEMS}

The main intention of this section is to describe the proposed approach along with the outline of the work.

\subsection{OUTLINE OF THE WORK}

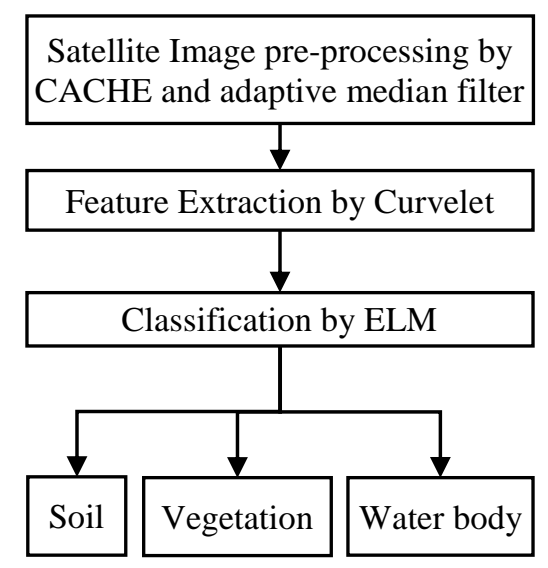

Fig.1. General Flow Diagram of the proposed approach

The main objective of this work is to classify between soil, vegetation and water bodies. This kind of classification system is beneficial for agricultural applications, which lets the user to know about the availability of water source and the vegetation over a particular area. To achieve the research goal, this work depends on three important phases such as pre-processing, feature extraction and classification. The initial phase is meant for noise removal and contrast enhancement, which is achieved by adaptive mean filter and CLAHE technique. The pre-processed images are suitable for the process of feature extraction. The texture features are extracted by applying curvelet transform to avail curvelet moments. Finally, the classification is performed by ELM. The general flow of the proposed work is presented in Fig.1.

Basically, the proposed work is executed in two stages which are training and testing. The training stage imparts the knowledge to the machine to make it eligible for classifying between soil, vegetation and water bodies. The ELM is trained by the feature sets being extracted. The testing stage is meant for passing a query image, such that the system can classify between the categories, by applying the previously gained knowledge.

\subsection{SATELLITE IMAGE PREPROCESSING}

The image pre-processing is the most fundamental step of any image processing application. The main goal of this phase is to eliminate the unwanted information and to improve the quality of an image. The images are pre-processed, as per the requirement of the application. This work aims to remove the noise and enhance the quality of an image. This work employs adaptive median filter and CLAHE technique for noise removal and contrast enhancement respectively. The pre-processed images are shown in Fig.2.

The reason for the employment of adaptive median filter is that it removes the noise without disturbing the edges. The adaptive median filter identifies the noisy pixel by comparing the current pixel with the neighborhood pixels. In case of the detection of any unmatched pixel with respect to the neighborhood pixels, the value of unmatched pixel is altered with the median value of the neighborhood pixels. The merit of this filter is that the neighborhood window size is not fixed.
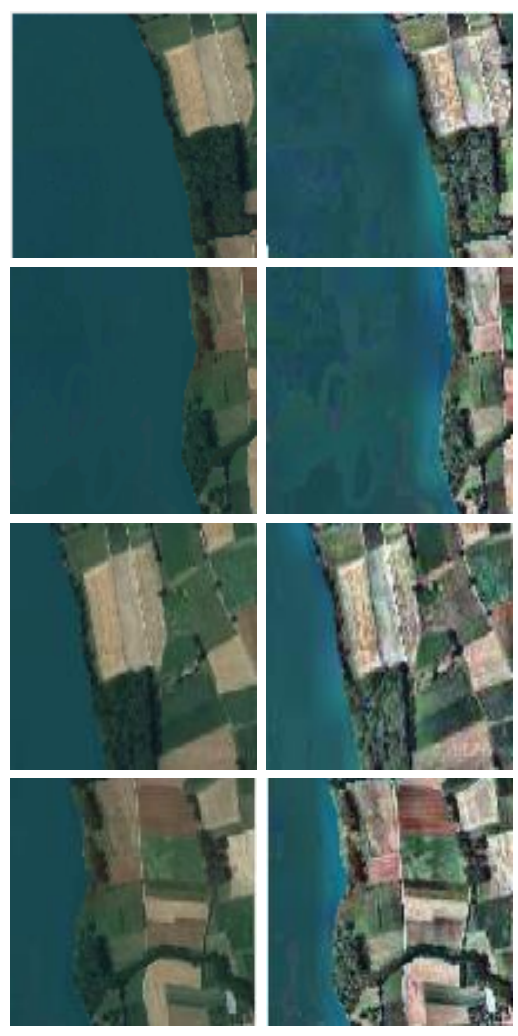

Fig.2. Sample pre-processed images

The pseudocode of adaptive median filter is as follows.

\section{Pseudocode 1: Satellite Image Pre-processing}

//Noise Removal

Step 1: Input: Noisy image

Step 2: Output: Denoised image

Step 3: Begin 
Step 4: Read image

Step 5: Compute the valid area limits of the filter;

Step 6: Check the values of the pixels in the window;

Step 7: Compute the ROI min, max and median values of the pixel;

Step 8: if med $>\min \& \&$ med $<\max$

Step 9: if centerpixel $\leq$ min $\|$ centerpixel $\geq \max$

Step 10: image $($ rows, cols $)=$ rmed ;

Step 11: end;

Step 12: end;

Step 13: end;

//Contrast enhancement

Step 14: Begin

Step 15: Divide images into tiles;

Step 16: For each tile

Step 17: Apply contrast transformation;

Step 18: Combine the tiles by bi-linear interpolation;

Step 19: End;

After the process of noise removal, the contrast of the images is intended to be improved by CLAHE technique. The CLAHE technique enhances the contrast of an image locally by managing the noise amplification.

\subsection{FEATURE EXTRACTION BY CURVELET}

This work employs curvelet transform, owing to its multiscale image depiction. Curvelet is an enhancement of wavelets and it beats the performance of wavelets [9]-[11]. The preprocessed images are decomposed into four levels followed by which the curvelet moments are extracted. The concept of moments is very popular in the area of image analysis, image processing and pattern recognition [12] [13]. The curvelet moments are computed as follows.

Consider $P=\left(P_{i}\right)_{1 \leq i \leq K}$ as the $K$ curvelet coefficient distribution. The following equation denotes the statistical curvelet moment $C_{o}$, which is of order $o$. The first moment is mean and is computed by,

$$
M=\frac{1}{K} \sum_{i=1}^{K}\left(P_{i}\right)^{o}
$$

The second moment is the variance, which indicates the variance of the mean value. The variance is computed by the following equation

$$
V=\frac{1}{K} \sum_{i=1}^{K}\left(P_{i}-M\right)^{o}
$$

The third moment is the skewness, which determines the degree of asymmetry over the mean value. Skewness is computed by Eq.(3)

$$
S=\frac{M^{3}}{V^{1.5}}
$$

The fourth moment kurtosis determines the peak or flat value of the intensity distribution by taking the mean value into account. The kurtosis is computed by the following equation.

$$
K=\frac{M^{4}}{V^{2}}-3
$$

The above mentioned four moment descriptors such as mean, variance, skewness and kurtosis are extracted from all the images, so as to form the curvelet moments. The maximum order being utilized by this work is four. The reason is that when the moment order goes high, it introduces computational complexity and instability. The feature vector is formed by the following equation

$$
F_{v}\left(f\left(x_{i}, y_{j}\right)\right)=\llbracket C M_{R C}\left(f\left(x_{i}, y_{j}\right)\right) \rrbracket
$$

During the training process, the feature vectors are formed and stored for future reference. This feature vector plays a vital role in the process of classification. The ELM is trained with the feature vector, which helps in classifying between the soil, vegetation and water bodies. The next section presents the ELM classification phase.

\subsection{ELM CLASSIFICATION}

ELM is employed for the purpose of classification, as it is proven to be the swiftly and reliable classifier [14]. During the training process, the ELM is trained with the knowledge gained from the feature extraction phase through the feature vectors. This prior knowledge helps in classifying between different categories The RBF kernel is utilized for ELM classification, the working of ELM classifier is as follows:

Let $X$ be the training samples represented as $\left(a_{i}, b_{i}\right)$ here $a_{i}=$ $\left[a_{i 1}, a_{i 2}, \ldots, a_{i s}\right]^{q} \in \mathrm{Im}^{s}$, where $n$ is the dimension of the training representatives. $b_{i}=\left[b_{i 1}, b_{i 2}, \ldots, b_{i t}\right]^{q} \in \operatorname{Im}^{t}$ indicates the $i^{\text {th }}$ class label of dimension $t$. Here $t$ is the number of classes, (its value is 3 in this case). A Single hidden Layer Feed-Forward Neural Network (SLFN) is built by an activation function $\operatorname{act}(x)$ and $R$ neurons. The activation function is denoted by,

$$
\sum_{i=1}^{R} \beta_{i} \cdot \operatorname{act}\left(w t_{i} \cdot a_{i}+e_{i}\right)=b_{i} ; i=1,2, \ldots, n
$$

In the Eq.(6), $w t_{i}$ is the weight of the feature vector, $e_{i}$ is the bias of the $i^{\text {th }}$ hidden neuron. Consider $H d_{l}$ as the ELM's hidden layer output matrix, where the $i^{\text {th }}$ column of $H d_{l}$ indicates that the $i^{\text {th }}$ hidden neurons output vector by considering the inputs $a_{i 1}$, $a_{i 2}, \ldots, a_{i n}$

$$
\begin{gathered}
H d_{l}=\left[\begin{array}{ccc}
a c t\left(w t_{1} \cdot a_{1}+e_{i}\right) & \ldots & \operatorname{act}\left(w t_{v} \cdot a_{1}+e_{G}\right) \\
\vdots & \vdots & \vdots \\
\operatorname{act}\left(w t_{1} \cdot a_{n}+e_{i}\right) & \cdots & \operatorname{act}\left(w t_{v} \cdot a_{n}+e_{G}\right)
\end{array}\right] \\
\beta=\left[\begin{array}{c}
\beta_{1}^{q} \\
\vdots \\
\beta_{G}^{q}
\end{array}\right] \\
B=\left[\begin{array}{c}
b_{1}^{T} \\
\vdots \\
b_{n}^{T}
\end{array}\right]
\end{gathered}
$$

The matrix form is represented as

$$
H d_{l} \beta=B
$$


The output samples are calculated by norm least-square solution, and the equation is given as follows

$$
\beta=H d_{l}^{\dagger} B
$$

where, $H L^{\dagger}$ is the $H L^{\prime}$ 's Moore-Penrose generalized inverse. The ELM training phase is achieved by computing Eq.(11). During the testing phase, the output matrices are calculated and added together, in order to detect the greatest value against the row. The output matrix is calculated by

$$
b_{\text {testing }}(z)=H d_{\text {ltesting }}(z) \times \beta_{z}
$$

This work fixes the value of $z$ as 12 , as it generates the most feasible results. The performance of the proposed approach starts to degrade, when the value of $z$ goes beyond 12 .

\section{EXPERIMENTAL RESULTS}

This work takes the images of the Aeugst am Albis of Switzerland into account for the purpose of image classification. The images being considered for this research lie between 47.268703 latitude and 8.491021 longitudes. The GPS coordinates of the image is $47^{\circ} 16^{\prime} 7.3308^{\prime \prime} \mathrm{N}$ and $8^{\circ} 29^{\prime} 27.6756^{\prime \prime} \mathrm{E}$. This section analyses the performance of the proposed approach by varying the feature extraction and classification techniques in terms of accuracy, sensitivity and specificity. The proposed approach is tested by considering the satellite images downloaded from the quickbird site [8]. The experimentation is done in the Matlab environment. The sample classification results are shown in the Fig.3(a)-Fig.3(d) and Fig.3(e)-Fig.3(h). In which, the pink color denotes water bodies, green and red colors represent vegetation and soil respectively. The classification performance of the proposed approach is evaluated by comparing the attained results with the ground truth images and the performance is evaluated as follows.

Classification accuracy is the most important parameter for any classification algorithm. The efficiency of the classification depends on the effectiveness of the features being extracted. The accuracy of the classification algorithm is computed by the following equation.

$$
a c_{\text {rate }}=\frac{T P+T N}{T P+T N+F P+F N} \times 100
$$

Sensitivity and specificity are other important measures that could rate the performance of the classification algorithm. Sensitivity is the measure which is the rate of correctly classified images to the sum of images that are correctly classified as positive and wrongly classified as negative. Specificity is measured by the ratio of the sum of images that are correctly classified as negative to the sum of images that are incorrectly classified as positive and correctly classified as negative. The sensitivity and specificity are represented as follows.

$$
\begin{aligned}
& \text { sens }_{\text {rate }}=\frac{T P}{T P+F N} \times 100 \\
& \text { spec }_{\text {rate }}=\frac{T N}{F P+T N} \times 100
\end{aligned}
$$

where, $T P$ is the count of images that are correctly classified with respect to the class and $T N$ is the count of images that are correctly classified as these images do not belong to a particular class. FP is the count of images that are wrongly classified as these images belong to a particular class and $F N$ are the count of images that are misclassified as the images do not belong to a specific class. The accuracy, sensitivity and specificity are measured by varying the feature extraction techniques and classifiers (RVM, SVM, ELM). They are presented in Fig.4 and Fig.5.

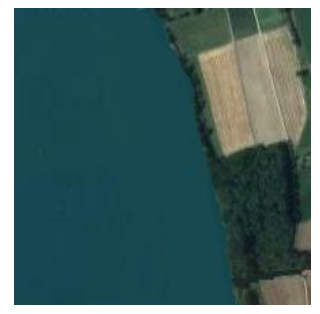

(a)

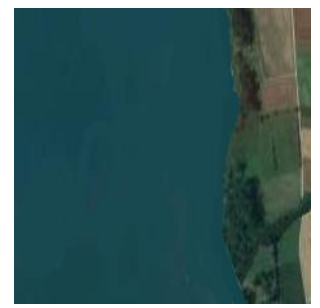

(b)

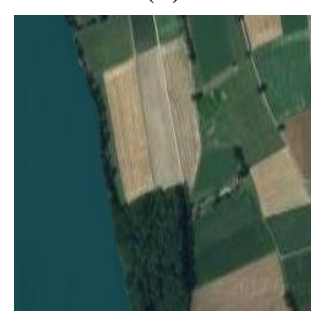

(c)

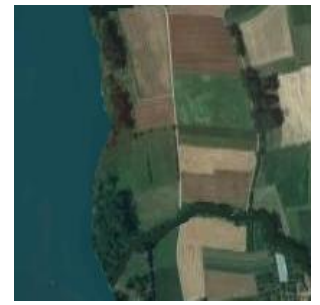

(d)

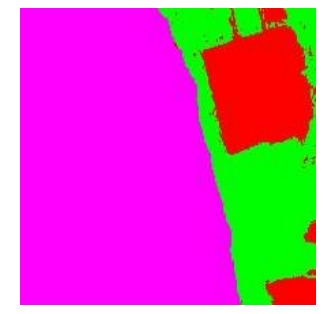

(e)

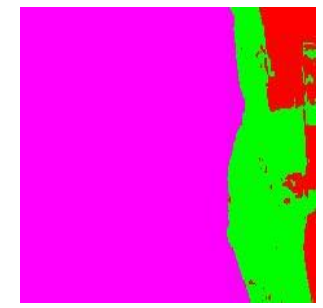

(f)

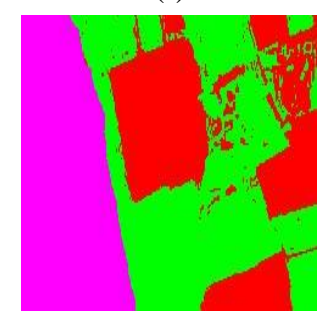

(g)

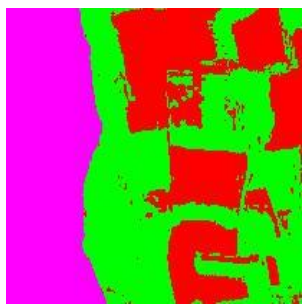

(h)
Fig.3 (a)-(d) Original images (e)-(h) classified images

The performance of ELM is compared with other analogous classifiers such as Relevance Vector Machine (RVM) and Support Vector Machine (SVM). From the experimental results, the efficacy of the ELM is proven. Though the experimental outcome of SVM is comparable with ELM, yet ELM performs better. The maximum accuracy, sensitivity and specificity rates are achieved by ELM. From the experimental results, it is evident that the proposed satellite image classification algorithm performs better in terms of standard performance measures. 


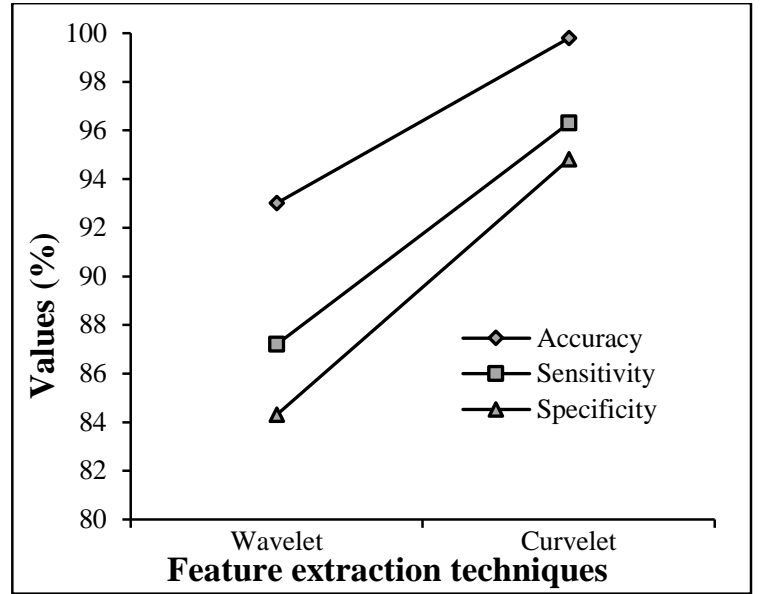

Fig.4. Analysis by varying feature extraction

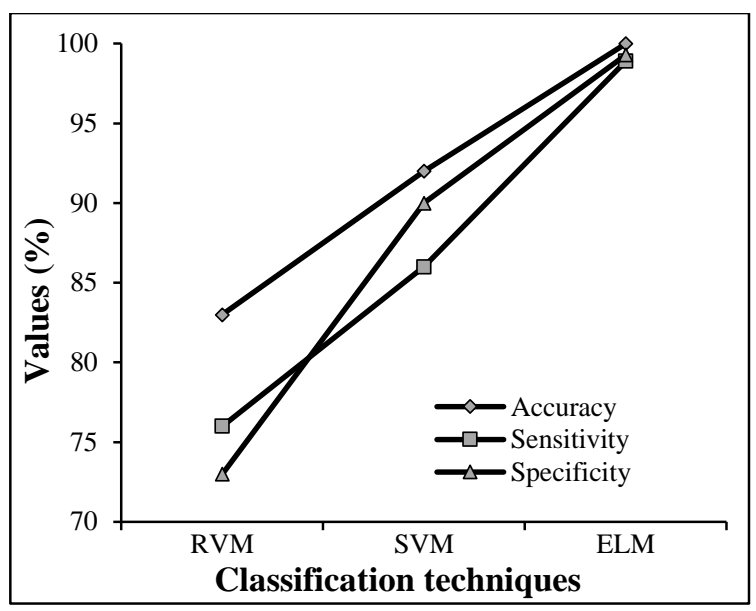

Fig.5. Analysis by varying the classifiers

\section{CONCLUSION}

This article proposes a curvelet based satellite image classification system for distinguishing between soil, vegetation and water bodies. Three significant phases are involved in the proposed work, which are image pre-processing, feature extraction and classification. The image pre-processing phase is achieved by adaptive median filter and CLAHE technique. The features of the pre-processed images are extracted by the application of curvelet. Finally, ELM is employed as the classifier to distinguish between soil, vegetation and water bodies. The performance of the proposed approach is observed to be satisfactory. In future, this work plans to include the feature selection techniques for minimizing the execution time of the system. Besides this, we plan to propose a vegetation classification system to classify between the crops.

\section{REFERENCES}

[1] P. Mather and B. Tso, "Classification Methods for Remotely Sensed Data", $2^{\text {nd }}$ Edition, CRC Press, 2009.

[2] Sunitha Abburu and Suresh Babu Golla, "Satellite Image Classification Methods and Techniques: A Review", International Journal of Computer Applications, Vol. 119, No. 8, pp. 20-25, 2015

[3] Pooja Kamavisdar, Sonam Saluja and Sonu Agrawal, "A Survey on Image Classification Approaches and Techniques", International Journal of Advanced Research in Computer and Communication Engineering, Vol. 2, No. 1, pp. 1005-1009, 2013.

[4] Shabnam Jabari and Yun Zhang, "Very High Resolution Satellite Image Classification using Fuzzy Rule-Based Systems", Algorithms, Vol. 6, No. 4, pp. 762-781, 2013.

[5] M. Chandrakala and R. Amsaveni, "Classification of Remote Sensing Image Areas using Surf Features and Latent Dirichlet Allocation", International Journal of Advanced Research in Computer Science and Software Engineering, Vol. 3, No. 9, pp. 178-182, 2013.

[6] S. Muhammad, G. Aziz, N. Aneela and S. Muhammad, "Classification by Object Recognition in Satellite Images by using Data Mining", Proceedings of International Conference on World Congress on Engineering, pp. 1-6, 2012.

[7] A. Selim, "Spatial Techniques for Image Classification", CRC Press, 2006.

[8] Satellite Imaging Corporation, Available at: www.satimagingcorp.com

[9] E. Candes and D. Donoho, "Curvelets: A Surprisingly Effective Non-Adaptive Representation for Objects with Edges", Proceedings of IEEE International Conference on Image Processing, pp. 105-120, 2000.

[10] L. Li, X. Zhanga, H. Zhanga, X. Hea and M. Xua, "Feature Extraction of Non-Stochastic Surfaces using Curvelets", Precision Engineering, Vol. 39, No. 2, pp. 212-219, 2015.

[11] L. Dettori and L. Semler, "A Comparison of Wavelet, Ridgelet, and Curvelet-based Texture Classification Algorithms in Computed Tomography", Computers in Biology and Medicine, Vol. 37, No. 4, pp. 486-498, 2007.

[12] F. Murtagh and J. Starck, "Wavelet and Curvelet Moments for Image Classification: Application to Aggregate Mixture Grading", Pattern Recognition Letters, Vol. 29, No. 10, pp. 1557-1564, 2008.

[13] C. Dalfo, M. Fiol and E. Garriga, "Moments in Graphs", Discrete Applied Mathematics, Vol. 161, No. 6, pp. 768777, 2013.

[14] Guang-Bin Huang, Hongming Zhou, Xiaojian Ding and Rui Zhang, "Extreme Learning Machine for Regression and Multiclass Classification", IEEE Transactions on Systems, Man and Cybernetics-Part B, Vol. 42, No. 2, pp. 513-529, 2012. 